\title{
Development of an Augmented Reality Concept for Icebreaker Assistance and Convoy Operations
}

\author{
Synne Frydenberg ${ }^{1, *}$, Katie Aylward ${ }^{2}{ }^{\oplus}$, Kjetil Nordby ${ }^{1}$ and Jon Olav H. Eikenes ${ }^{3}$ \\ 1 The Oslo School of Architecture and Design, 0175 Oslo, Norway; kjetil.nordby@aho.no \\ 2 Chalmers University of Technology, 41296 Göteborg, Sweden; katie.aylward@chalmers.se \\ 3 Finn.no, 0159 Oslo, Norway; jonolav.eikenes@gmail.com \\ * Correspondence: synne.g.frydenberg@aho.no; Tel.: +47-97613623
}

Citation: Frydenberg, S.; Aylward,

K.; Nordby, K.; Eikenes, J.O.H.

Development of an Augmented

Reality Concept for Icebreaker

Assistance and Convoy Operations. J.

Mar. Sci. Eng. 2021, 9, 996. https://

doi.org/10.3390/jmse9090996

Academic Editor: Apostolos

Papanikolaou

Received: 13 August 2021

Accepted: 6 September 2021

Published: 13 September 2021

Publisher's Note: MDPI stays neutral with regard to jurisdictional claims in published maps and institutional affiliations.

Copyright: (C) 2021 by the authors. Licensee MDPI, Basel, Switzerland. This article is an open access article distributed under the terms and conditions of the Creative Commons Attribution (CC BY) license (https:/ / creativecommons.org/licenses/by/ $4.0 /)$.

\begin{abstract}
A vessel convoy is a complex and high-risk operation completed during icebreaking operations in the Arctic. Icebreaker navigators need to continuously communicate with their crew while monitoring information such as speed, heading, and distance between vessels in the convoy. This paper presents an augmented reality user interface concept, which aims to support navigators by improving oversight and safety during convoy operations. The concept demonstrates how augmented reality can help to realize a situated user interface that adapts to user's physical and operational contexts. The concept was developed through a human-centered design process and tested through a virtual reality simulator in a usability study involving seven mariners. The results suggest that augmented reality has the potential to improve the safety of convoy operations by integrating distributed information with heads-up access to operation-critical information. However, the user interface concept is still novel, and further work is needed to develop the concept and safely integrate augmented reality into maritime operations.
\end{abstract}

Keywords: augmented reality; icebreaker assistance; convoy operations; navigation; human-centered design; user interface design; virtual reality-reconstructed operation scenarios; the Arctic

\section{Introduction}

Augmented reality (AR) is an emerging technology that superimposes information on top of a person's view anywhere in the environment. AR technologies can support navigation and operation on a ship's bridge by combining the real world outside the vessel with data from bridge systems [1]. However, the possibilities and premises for designing AR applications in the maritime sector are demanding to explore [2]. The spatial, physical, and temporal possibilities of this technology challenge the current practice within the field of interaction design, which up until now has been mostly screen-based [3]. In this early phase of building knowledge of how to design for AR, our aim is to develop design examples of AR applications in order to build useful design frameworks for AR which can potentially support practicing interaction designers [4]. The background motivation for designing a software application for icebreaker assistance and convoy mode derives from our design-driven field research at sea [5], where our interviews and observations with navigators demonstrated that the premises for icebreaker assistance had considerable potential for the improvement of safety for both the icebreaking vessel and the assisted vessels during these critical operations. Based on these findings, we have continued our human-centered approach with the aim of supporting the navigator to achieve enhanced control and overview during icebreaker assistance operations [6].

The AR technology is becoming increasingly viable for maritime use, particularly when information needs to be integrated during complex operations, for example, in the Arctic [7]. Maritime traffic in the Arctic is increasing, as previously unavailable shipping routes are becoming more accessible. The naturally harsh operating environment in the high north offers challenges for many shipping sectors and can have a remarkable effect on 
voyages. The lack of important data, such as weather reports, ice conditions, latitude errors, and varying global navigation satellite system positioning errors, makes navigational activities highly demanding, especially for navigators with little experience sailing in Arctic regions [8]. However, accurate sensor data are not enough—available data need to be presented in a way that supports mariners efficiently during demanding conditions and stressful operations. Mistakes can have major consequences, and help is often out of reach in the high north [9]. Therefore, ship bridges sailing in Arctic waters should be equipped with systems that support navigation and operations in ice.

Increased interest in and usage of passages in the high north requires an extended focus on facilitating and supporting safe operations. Icebreaker assistance in various forms is an important part of this situation, given that navigation in Arctic waters is regularly dependent on such support [10].

Icebreaker assistance is commonly divided into five specific operation types [11]: (1) vessel escorting operations involve an icebreaker breaking a channel of ice through which another vessel can follow at a particular distance, (2) breaking loose operations involve an icebreaker sailing near a vessel stuck in ice in order to release ice pressure, (3) convoy operations involve an escorting operation containing more than one vessel following, (4) double convoy operations involve two icebreakers sailing offset aligned in order to break a wider path for a broad vessel, and (5) towing operations involve towing the assisted vessel while breaking the path due to heavy ice pressure or a large amount of slush ice.

In ice-filled waters, such as the Baltic Sea, ship collisions are one of the most frequent accidents occurring during winter [12]. Convoys are one of the most dangerous and unpredictable operations during the wintertime [13]. The most crucial risk is represented by collisions between assisted vessels or between an assisted vessel and an icebreaker [12]. Relatively high speed and proper distance must be maintained during the entire operation [13]. Although crews on the icebreaker vessel recommend a certain speed and distance between assisted vessels, crews on the assisted vessels are responsible for maintaining this advice. This work can be challenging to both the icebreaker and the assisting vessel because it requires constant attention. A minor misunderstanding in communication or an operational variation can cause the vessel to deviate from the set speed and distance. Too little distance between two ships increases the risk of collision, whereas too much distance involves an increased risk of being obstructed by slush or stuck in ice [13].

According to navigators on icebreakers, experience-based rules of thumb for leading operations in a safe way are important [11]. They also emphasize the importance of systematic knowledge about operational characteristics and ship domains, including safe speed and distance according to the specific area. However, unpublished reports from field studies on icebreaker vessels conducted in the Safe Maritime Operations Under Extreme Conditions: The Arctic Case (SEDNA) project indicate that these requirements are not met optimally in the current bridge systems; navigators experience a lack of overview at several levels during icebreaker assistance operations. First, an overview of the assisted vessels is insufficient in digital bridge systems, which lack detailed key information. Second, in the physical overview outside the vessel, monitoring can be limited for other vessels during operations due to obstacles on the vessels and/or weather conditions, such as fog or darkness. Third, communication with the other vessels on very high frequency (VHF) radio may be unclear due to poor connection or language skills. A high situation awareness (SA) should be maintained for navigators leading icebreaker assisting operations by designing user interfaces (UIs) for bridge systems that take these specific user requirements into account.

The need for icebreaker assistance can arise from different situations, for instance, as a planned escort of a vessel with a lower ice class than recommended for the waters or rescuing a vessel stuck in the ice [13]. During an assisted convoy operation of several vessels, the icebreaker secures a safe passage by breaking ice at the front and leading the other vessels through the path at an appropriate distance and speed to their requested 
targets. The icebreaker's leading role requires careful monitoring of the other vessel's key information, as well as clear communication to safeguard the progress.

Today, external communication between vessels during convoys is transmitted through VHF radios, where ships' bridge crews exchange orders and answers. Due to potentially unstable connections and poor language skills, such forms of communication can easily lead to misunderstandings $[13,14]$. During a convoy operation, a misunderstanding, such as missing an order to "lower the speed to X knots," can be fatal. The internal communication on the bridge, which is an important part of Bridge resource management (a tool for safety and error management, see [15]), is particularly exposed to noise and poor system overview with data sharing during icebreaking operations. The monitoring can be supported by data from other systems, such as an Electronic Chart Display and Information System (ECDIS) or an Automatic Identification System, as well as by visual monitoring. However, accuracy and the degree of relative details of current systems are inadequate for icebreaker assistance operations; furthermore, research on the problematics of positioning in the Arctic region concludes that complementary positioning methods are required to achieve an accuracy of fewer than three meters [16]. Examples of such positioning methods are laser-based position reference systems, such as SpotTrack [17] and Differential absolute and relative position sensors, which are a dynamic positioning reference system [18]. The accuracy of the positioning affects the input data and the possibilities for developing supportive UI representations for icebreaker navigators. By UI representation, we refer to the space where interaction between the user and the systems occurs, which is either physical, such as a keyboard; or digital, such as a screen; virtual, such as an AR UI. The UI representation should be fitted to the user's situation in order to provide a sufficient overview.

Good SA is important, and in this case refers to the navigator's capability to perceive and comprehend what is going on at the bridge, in the oceanscape, and on other vessels, and the ability to project future status [19]. To maintain good SA throughout the operation, navigators need options for monitoring other vessels' speed, distance, and relative value [20]. In addition, a navigator must be able to have a full overview ahead to operate their own vessel. In the current situation, navigators need to perform a considerable amount of integration work between systems to sort and interpret key information [21]. To meet this challenge, we suggest that a convoy UI should ideally be integrated to appear from the navigator's point of view, such as by using AR technologies.

In recent years, the development of AR technologies has been rapid and marketdriven, especially after the launch of head-mounted AR technologies, such as Microsoft Hololens [22]. The potential for AR in the maritime domain shows a promising outlook [23], and previous research has uncovered a range of suitable scenarios for its use on ship's bridges [3,4]. Navigator's SA and performance can be enhanced through the use of AR by functioning as an information support for navigating, regulating information overload, reducing integration work, and connecting digital and analogue information [3,23-26]. Nevertheless, research on how to involve users' needs in the design process of AR technology for the maritime domain remains limited [4]. Therefore, we argue the need to develop tools with relevant and specific data and flexible UIs that support navigators with precise information on icebreaker assistance operations, such as during convoy operations.

We approach this problem by asking the following research question: How can AR support navigators during convoy operations on ice? We answer the question by, firstly, demonstrating an example of how AR can be designed as a bridge application with the potential to support the navigator's SA during icebreaker operations. We describe and illustrate the UI concept with its functions and content. Secondly, we present a usability study of how end users have tested the application concept. The overarching approach for both the first and the second parts of this study is the application of contextual processing in a virtual reality (VR) simulator instead of on a real ship bridge [27]. This methodological approach for concept development and user testing is described in detail in the Materials and Methods section. We evaluate the concept and test, indicate limitations in the current UI concepts, and discuss further developments. 
In the SEDNA project, we have investigated how we can develop safe and efficient design concepts for AR-enhanced ship bridges through a user-centered approach. This approach involves mapping and analyzing current user needs. These methods affect how the usability of new design proposals can be evaluated and how the design frameworks we continuously iterate can be generalized for the design of new AR systems. This SEDNA research builds on and extends research from previous and ongoing research projects, such as the Onsite and Ulstein Bridge Concept and OpenBridge (OB). It connects to the OB [28], where an extensive open design system is developed to make the UI design consistent on a ship's bridges across vendors and equipment. This consistency implies preset rules for fonts, sizes, placement, appearance, colors, and, to some degree, logics for functions. Currently, the OB design system includes guidelines for screens and monitors of various sizes and qualities. However, expanding the OB design system to include AR-specific design guidelines is a relevant direction to examine further. We have used the foundation of the OB design system and further developed it to function for AR in this case study, designing better system UIs for icebreaker assistance operations.

The research is part of the EU project Safe Maritime Operations Under Extreme Conditions: The Arctic Case (SEDNA) and is one of several cases developed with the aim of improving efficiency and security for vessels operating in ice-filled waters.

In the following section, we describe the method for developing and evaluating our UI concept, the results, and the challenges and advantages of augmenting an existing design system through this case study.

\section{Materials and Methods}

To investigate the potential of AR, we needed to both explore possible solutions by designing examples of application UIs and evaluate the usefulness of those solutions by conducting usability testing. Therefore, our method was twofold: the first part of the approach involved design of the AR application while the second part contained the usability testing (Figure 1). We used two different contextual approaches for our methods during the study, field studies in real context during the first phase of Method 1, then what we define as virtual reality-reconstructed operation scenarios (VRROS) (described in the following paragraphs) during the last part of Method 1 and during the whole of method 2 (Figure 1). In the following, we will describe in detail these two methods and the two different contextual approaches and how they are interlinked.



Figure 1. The model illustrates the twofold nature of the methods in the study, first in the left bottom square, design of AR application, followed by the right bottom square, usability testing of the AR application. On the top of the model are two blue arrows which represents the contextual approach in the two methods and the timeline of the process. The process is divided into a shorter part of field studies in the beginning of Method 1 and a longer part of virtual reality-reconstructed operation scenarios (VRROS) during both Method 1 and 2. 
In Method 1, we used a human-centered design approach involving users in a way that emphasizes the human perspective throughout the research and design process [6]. For interaction design processes, creating products and services that enable users to achieve their goals in the best way possible is the main purpose [29]. In our case, supporting good SA is one of the goals. Therefore, many of the techniques and methods used for designing digital concepts, such as a bridge application, requires distinct understanding of both the use situation and the design situation [30]. By implementing two different contextual approaches throughout the study phase called Method 1 (Figure 1), we have maintained the important awareness of and distinction between the use situation and the design situation. In the following section we will describe the techniques and sub methods for design (Method 1) in this study.

The first part of our design phase was carried out in the contextual approach of field research expeditions on board several icebreakers operating and safeguarding different areas of Arctic and Baltic waters; our design process was undertaken in several stages of the project $[2,3]$. We used well-known field research methods, such as participatory observation [31] and co-design [32], and explored how methods and techniques for collecting quantitative data, such as eye-tracking data [33], can be used qualitatively as a digital underlay for sketching in AR and forms of rapid AR testing and prototyping [2,3]. The data captured in the field research context resulted not only in familiarization and deeper insights into the users and user situations, but also in testable ideas and concepts that were further developed in the lab.

The second part of the design phase and the usability study needed to be completed in our labs, mainly due to strict travel restriction and limited access during COVID-19 in 2020. However, UIs for ships' bridges should be designed with an emphasis on the situation in which they will be used [4]. For most designers and researchers, the Arctic maritime context is an unfamiliar setting with hard-to-reach users [34] and a demanding context to access and work in overtime [9]. In order to continuously engage with this context after the initial field studies, our process contained a continuation of the previous fieldwork onboard icebreakers within a lab setting by using what we call VRROS (Figure 2) [27]. The VRROS represent the contextual approach for our second phase of design (Method 1) and for the whole process of usability testing (method 2). The VRROS have been developed in our lab by recreating icebreaker operations in a VR-enabled simulator. The operational scenarios were designed in collaboration with and verified by usability experts and expert end users. The VRROS proved to be ideal for design development of UI concepts in the form of visual prototypes embedded in and immersed with the VR environment in the VRROS. This allowed us to rapidly test and evaluate design ideas and concepts according to a simulation of existing premises on a vessel $[27,35]$. In addition, the VRROS approach allowed us to maintain involvement with users, designers, and human factor (HF) specialists throughout the design and testing process.

In this study, the chosen VRROS consist of a vessel stuck in ice, requiring assistance from an icebreaker and a resulting convoy operation (Figure 2). The scenario contains a course of action from a defined start and an end with given premises, such as operations, targets, equipment, and weather conditions. A precise scenario timeline was described to achieve a high degree of realism. The detailed maritime context found in this scenario was then recreated in VR. The VR simulator is built with the Unity game engine, which can produce a realistic experience of contextual factors, such as light, weather, and icebergs, in parallel with the scenario timeline. This allows designers and researchers in the lab to test how ideas and concepts for AR technology can be applied at an early stage. The VR simulator functions as a tool for concept development and as a setting for future experimental testing of the concept. 


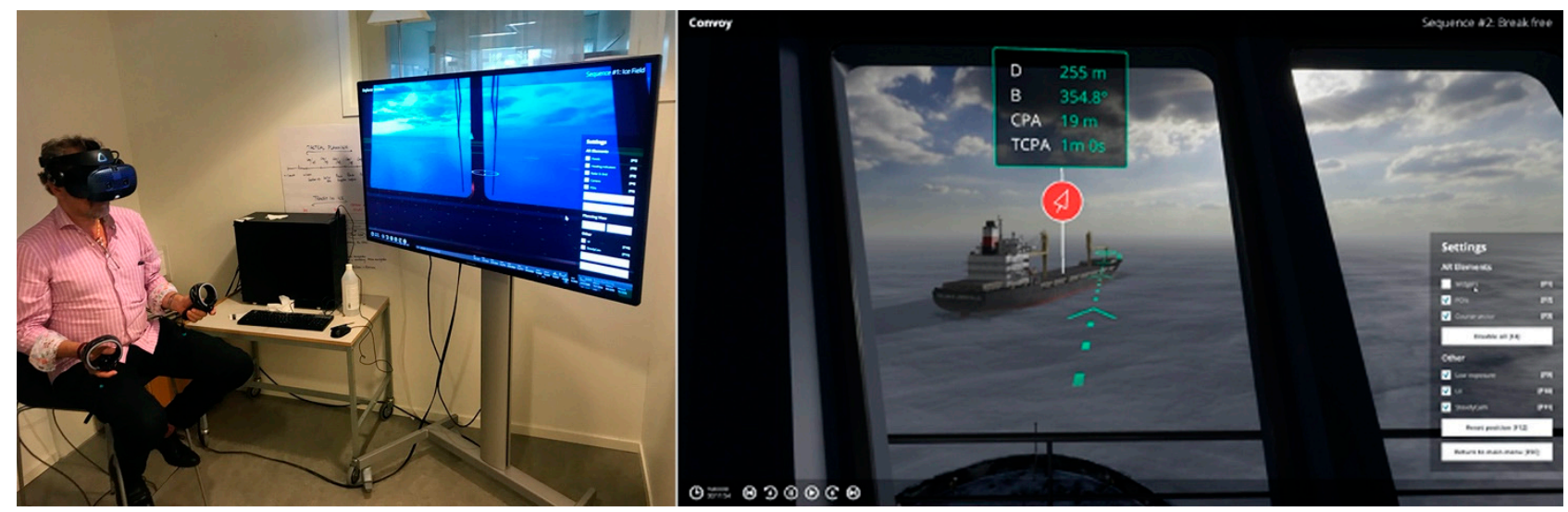

Figure 2. To the left: the setup of the virtual reality-reconstructed operation scenarios (VRROS) seen from an exocentric perspective which represents the test facilitators who can monitor both the user's behavior in the room and what the user sees in the VR headset on the big screen. To the right: a screenshot of representing the egocentric perspective of what the user sees within the VRROS.

The VRROS (Figure 2) included three vessels: two cargo vessels and one icebreaker. The VR user was situated on the icebreaker ship's bridge and had an overview of the existing console screens and the AR UIs. The vessel moved according to previously recorded bridge simulator data. Thus, the user could not control the vessel but could move freely around the bridge. The bridge systems were of modern standard, with consoles and large screens displaying ECDIS and radar interfaces designed according to OB design guidelines [28] in a dusk palette. The VR scene lasted for $67 \mathrm{~min}$, where the facilitator interface allowed the researcher to shift among five different chapters of the scenario timeline: (1) transit in ice, (2) break free, (3) convoy, (4) stop, and (5) loop back.

Designing for hard-to-reach environments by implementing and testing VRROS is a novel design approach. Through our studies, we were therefore simultaneously researching methodological potential. Some of the advantages were that the VRROS enabled us to isolate and concretize these separate parts of the scenario, which, from a technical perspective, could be defined as situations. Various premises, such as daylight, movement, and level of stress, could be set to occur during different phases of the operation within the scenarios. This allowed us to test adjustments of the AR information panes according to the current situation. Our aim is to develop designs for situational adaptation, which we define as UIs with which the user experiences meaningful interactions according to the situation. By developing VRROS, we can generalize a set of situations in which we can test several UI concepts, including a variety of situational adaptations, to explore how the interaction can be experienced as situated as possible. In this study, we investigated this potential by developing more operation-dedicated and situated UIs for monitoring and communicating with other vessels' icebreaker assistance operations.

The current AR hardware has optical challenges when transmitting graphics in bright environments. We demonstrated that some of these challenges can be reduced by sunscreen [35]. As technology progresses, we expect that the optical challenges will be remarkably reduced, and therefore we left the optical performance of AR technology outside the scope of this article. As a result, the figures in this article, which illustrate the AR concepts, are not optically correct but are rendered to illustrate the UI structure and functionality, given acceptable AR performance for a given context.

In this paper, we specifically describe the design proposal we developed for our own icebreaker assistance software application, containing key detailed information in strategically placed locations, filling the existing gaps in design. We focused on developing a specified mode for the application to be used during convoy operations. We developed several UI variations of the application with situational adaptations according to strategic placement in line with users' points of view, contents, functions required, and 
daylight modes. In the following section, we describe how we evaluated the usability of the application.

As part of Method 2, representing the usability study of the design concept is described as follows (Figure 1). Recruitment, which consists of purposive sampling, also known as judgment sampling, is a non-random technique used when a researcher needs participants to have certain qualities, skills, knowledge, or experience [36]. Purposive sampling was used to recruit participants, primarily through word of mouth, who had achieved Master Mariner certification at some point in their career and had recent experience with navigational equipment either through simulations or real life. The participants were not required to have any experience in ice navigation as this was not deemed necessary by the research team. The original plan for user testing was to recruit both fourth-year Master Mariner students and experienced navigators from Chalmers University of Technology and local shipping companies. However, the COVID-19 pandemic affected the ability to recruit and test the desired sample. The participants were therefore primarily recruited internally at Chalmers University of Technology.

The ethical considerations in this study are described in the following procedure. When the participants arrived at the test lab, they were provided with information about the SEDNA project and a brief description of the test protocol. The participants were also briefed about the potential risks related to VR use, including dizziness, nausea, and eye fatigue, and were told they could stop the test at any point. Each participant was given a unique ID number, which was used throughout the test to ensure confidentiality.

Based on the demographic data, the seven users who evaluated the scenario were professional mariners. All the participants were Swedish males. One participant was 25-34 years old, one was 35-44 years old, and the remaining five were $44-54$ years old. Six participants were employed by Chalmers University of Technology, three of which were Chalmers simulator instructors. One participant was currently serving onboard and was not associated with Chalmers. All participants were considered subject matter experts in maritime navigation. In addition to demographic information, the participants were asked about their previous experiences using VR. They reported that they either had no experience at all with VR systems (four responses) or little experience using VR systems (three responses).

The equipment was set up in a VR/AR test lab located at Chalmers University. The VR hardware used in the testing was a powerful PC equipped with a GeForce 2080Ti graphic card. The VR headset used in the test was the HTC Vive Cosmos. Additional equipment required to run the usability test included a video camera, an iPad, and a large TV screen. The tools used to communicate and share information between $\mathrm{AHO}$ and Chalmers included Box, a VR scenario developer, Zoom, TeamViewer, and Skype.

The procedures are described as follows. A pilot study was completed prior to the official data collection to ensure that the scenario was appropriate for subject matter expert evaluation. The pilot study consisted of a walkthrough of an example scenario to identify any potential issues with both the equipment and the test procedure. This provided the opportunity to obtain preliminary feedback on the AR features and helped to develop the final test questions for the participants.

Data collection was completed at Chalmers University of Technology in Gothenburg, Sweden, in October 2020, by two HF specialists. Both HF researchers were present throughout the data collection to observe, record participants' answers, and assist with the VR controls and maneuvering through the scenario. Participants were asked about simulator sickness (dizziness, feeling unwell, nausea) multiple times throughout the testing period. No participants reported any symptoms of sickness at any point. Once consent and familiarization were completed, the participant completed a tablet-based demographic pre-test questionnaire. When finished, the researcher provided the following instructions to the user:

The overall aim of this usability test is to evaluate specific AR solutions for enhancing situation awareness for navigators on the bridge. For this test, we recreated an icebreaker 
and convoy scenario in VR. The vessel follows a predefined path; in other words, you are not able to control the ship. We used vessel movement data from a simulator walkthrough with the accompanying RADAR and ECDIS screens. We want you to focus on how the different AR solutions may or may not enhance your awareness of what is going on, and specifically if/how the AR solutions could lower the risk of this specific operation. The scenario is divided into five "scenes". You will get to experience each scene for as long as you want before moving on to the next scene. You can also go back to a previous scene or replay a scene. The scenario in total will take approximately $60 \mathrm{~min}$. The quality and resolution of the VR headset are limited, and it might be hard to see some of the details. We assume that the AR quality will get much better, so try to imagine that the visual quality of the AR graphics is better than what you see in this test.

We want you to talk aloud during the exercise-describe what you see and how you experience/interpret what you see. We will also ask you specific questions about the different AR solutions; however, say whatever comes to mind as you experience the scenario. There is no right or wrong answer; we just want your honest opinion about the different solutions. We will ask you several times throughout the scenario if you feel sick, nauseous, or uncomfortable. We will stop the scenario if you feel unwell at any point.

The scenario began when a participant was seated and relaxed (Figure 2). The researchers allowed the participants to become comfortable on the virtual bridge. Most of the time, as they explored the bridge, a discussion about the UI concept emerged naturally. If the participants did not initiate a discussion themselves, they were prompted by the researcher. The UI concepts tested in the usability study are listed below (Table 1) and further described in the results.

Table 1. Elements of the UI concept and elements of feedback.

\begin{tabular}{|c|c|}
\hline UI Concept & Elements of Feedback \\
\hline Information pane (Figure $X-$ point to results photo) & \multirow{5}{*}{$\begin{array}{c}\text { Content of UI } \\
\text { Ability to interpret information } \\
\text { Size, placement, and color } \\
\text { Potential risks } \\
\text { Usefulness of the UI } \\
\text { Is any critical information missing? }\end{array}$} \\
\hline $\begin{array}{l}\text { Icebreaker assistance mode (Figure } X \text {-point to figure } \\
\text { in results—same for below concepts) }\end{array}$ & \\
\hline Convoy mode & \\
\hline Points of interest & \\
\hline Aft bridge UI & \\
\hline
\end{tabular}

A mixed-methods approach was adopted to collect and analyze the data. The focus was primarily on qualitative data obtained through a think-aloud protocol. Think-aloud methods allow participants to talk out loud or verbalize their thoughts while completing a specific task [37]. Both concurrent and retrospective verbal reports were completed throughout the data collection. The concurrent report involved the participants speaking out loud throughout the scenario as they encountered a specific AR solution. They spoke about what they saw and were probed with pre-determined specific questions about each AR feature if needed. The retrospective verbal report was completed post-scenario and required the participants to reflect on their experiences. They were asked to recall any additional comments or feedback about either the AR features or the VRROS experience.

In addition to the think-aloud protocol, HF specialists observed the participants throughout the entire scenario. The test setup allowed the researchers to have the same view as the participants through the TV screen while also observing their body language and movements (Figure 3). Any interesting observations were noted and added to the participants' testing sheets. 




Figure 3. VR/AR test setup at Chalmers University, showing one of the users wearing the VR headset and using portable controllers to interact and move around on the virtual ship bridge.

In terms of quantitative data, two questionnaires were developed using the software SurveyMonkey [38]. A basic demographic questionnaire was administered at the beginning of the test day, which captured the user's demographics, previous VR experience, and AR expectations. A post-test questionnaire was administered at the end of the test day, which asked specific questions about the usefulness of the AR technology and attempted to obtain a quantitative assessment of the user's overall experience using the technology.

The qualitative data were analyzed by two HF specialists who were present during the data collection. This process was followed to provide a cross-check system to ensure that the data were correctly interpreted. The data were first transcribed individually by each HF specialist, and then the transcripts were compared for consistencies or discrepancies. Once the researchers were satisfied that the transcripts reflected reality, both the individual participant transcripts and a summary of the comments about each AR widget were delivered to the design team. The questionnaire data were analyzed using basic Excel functions to summarize the demographics and the post-test questionnaire results.

\section{Results}

The results are presented in two separate parts. First, we present the results from the human-centered design process of developing a design example of the AR application Icebreaker assistance. Second, we present the results from the usability study, which assessed each AR UI mode, in addition to the users' perceptions of the overall usefulness of this application during icebreaking operations.

\subsection{Concept of Icebreaker Assistance and Convoy Mode}

We developed a software application called Icebreaker Assistance. The application is integrated into an information pane consisting of several other UI components used in combination during icebreaker operations, such as conning data and radar. The information pane can be integrated into the user's environment in several ways, for example, by fixing its position to a wall, on top of a window, or so that it can move around with the user. The application aims to support navigators in monitoring the status and relation to nearby vessels during icebreaker assistance. We envision the application to be relevant for use during several operations related to icebreaker assistance, such as when navigating in close distance to other vessels, such as during escort, towing, or breaking loose a vessel stuck in ice. The application can also be envisioned as a support in other situations apart from 
icebreaker assistance, such as rescue operations, inspection or other customs inspections at sea, or in entering/leaving trafficked harbors.

The application builds on the UI architecture of OB that describes how to design maritime graphical UIs [39] and current research expanding the OB framework into AR [4]. The illustrations in this article are represented in the palette for dusk light conditions. In the following section, we present the research process.

\subsubsection{Information Pane}

During icebreaker operations, the information pane is assembled by information components for the navigator's situated needs during icebreaker assistance, containing their own ship information, thrusters, map, and, highlighted in the red square, Icebreaker assistance (Figure 4). The Icebreaker Assistance application can consist of the information pane, however also has other individual appearances adapted to the situation, such as pinning of information to other vessels or as a translucent application mode placed close to windows on the bridge.

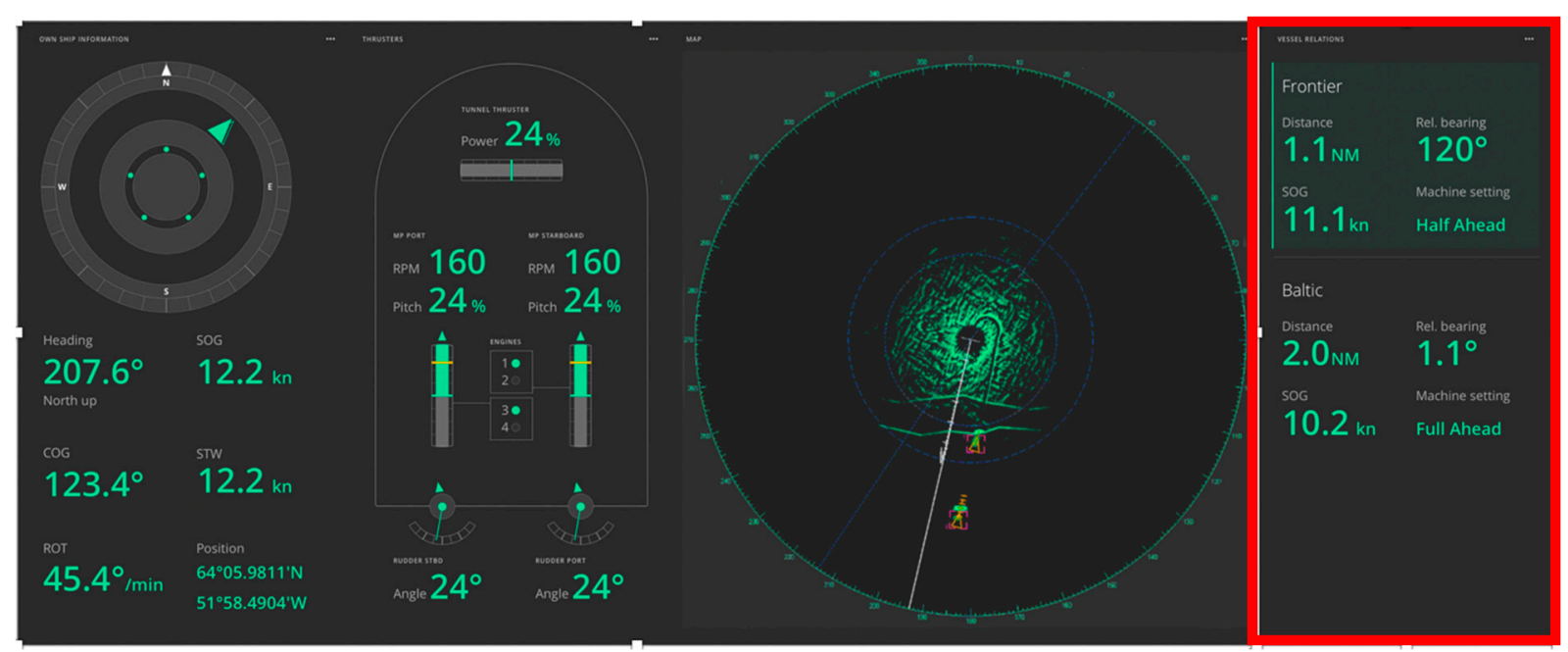

Figure 4. Information pane, consisting of an assembly of information components used during icebreaker assistance operations with the icebreaker assistance application marked in the red square.

\subsubsection{Icebreaker Assistance Mode and Convoy Mode}

In the general mode of the Icebreaker Assistance application, the names of related vessels are represented in addition to their current distance, relative bearing, speed over ground, and machine setting (Figure 4). Convoy mode is a specialized mode used during a convoy operation (Figure 5). The assets of convoy mode are useful in convoy operations of three or more vessels for monitoring the relations between vessels 2 and 3 (and possibly 3 and 4), where the vessels should have clear awareness of eventual alterations in speed and distance around other vessels. The specialized mode for convoys is activated when vessels enter a convoy operation and are ready to start (Figures 5 and 6).

The desired ideal speed and distance between the vessels can be set by the navigator. The distance between the vessels must not be too narrow due to the risk of collision; however, it must also not be too wide due to potentially heavy ice pressure that can force the ship to shrink.

Above are the participating vessels in the convoy, as represented by icons placed vertically in the existing order with vessel name to the left and speed over ground to the right. The distances between the vessels are represented by the distance to the left, relative speed over ground to the right, and are additionally supported by vertical arrows between the ships that indicate differences in speed. 


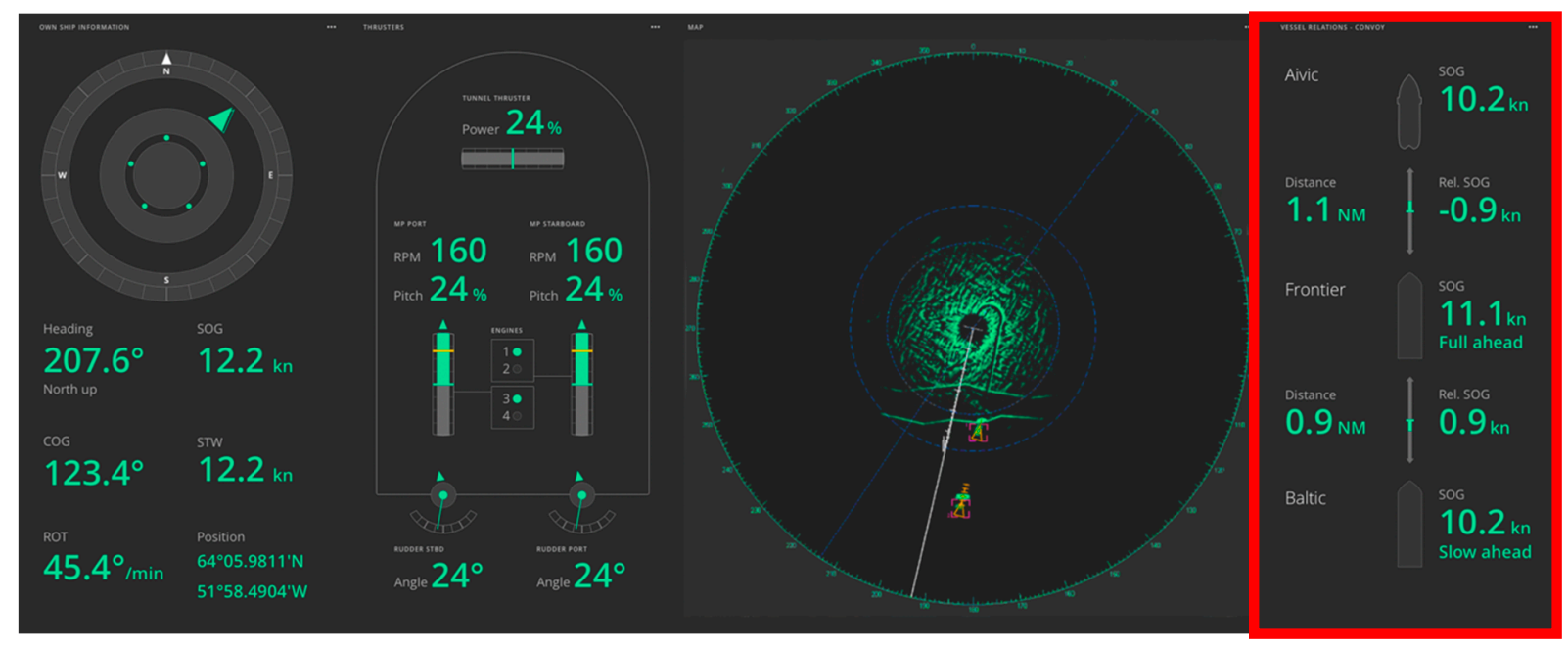

Figure 5. Information pane with convoy mode in the application Icebreaker Assistance marked in the red square.

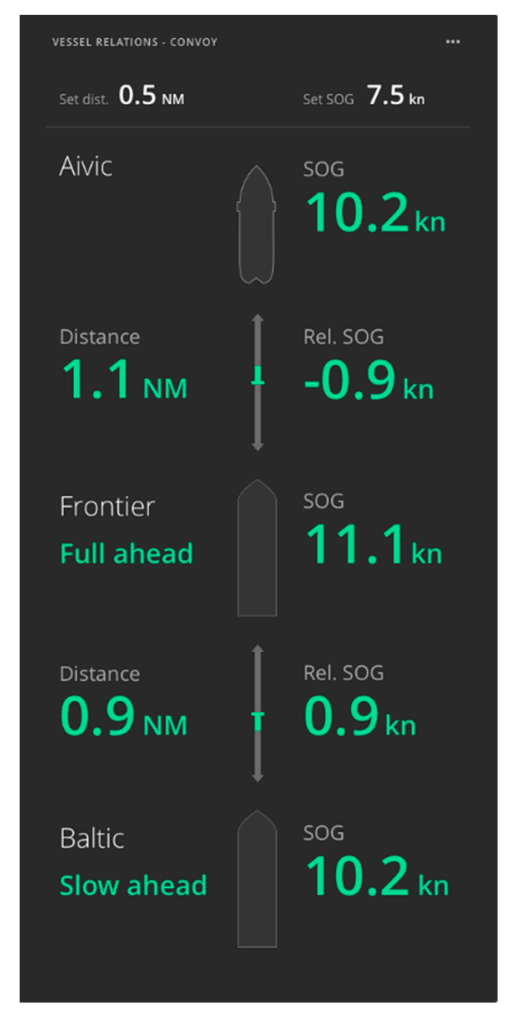

Figure 6. Close-up illustration of the Icebreaker Assistance application UI in convoy mode.

Engine settings for all vessels are included in the widget to allow navigators on the leading vessel to monitor other vessels' capacities and whether they are following instructions correctly. In Figure 5, the engine settings for the vessel Frontier are indicated by Full ahead, and, for the vessel Baltic, Slow ahead.

\subsubsection{Appearances and Placement}

The application consists of several representations with various options for appearance and placement, depending on the situation. For the usability test, we tested three different representations: points of interest (POIs), aft bridge information pane, and the large information pane. In the following section, we describe and illustrate the two additional representations. 
In icebreaker assistance mode, pinning information about POI, such as other vessels, in the outside view by the navigator to keep track of their critical information can be relevant. The pinning function of POIs represented by vessels nearby is illustrated in Figure 7. By default, a vessel is represented by a symbol in a circle, where the arrow indicates the heading of the other ship relative to the AR user's position. The symbol changes color from green to orange and then to red as the ships get close to each other to alert the user about the risk associated with close proximity between the vessels. When gazing at the symbol, more information appears: distance, (relative) bearing, closest point of approach (CPA), and time to CPA. Subsequently, the user can pin the information pane to keep it open or to add it to a widget, for example, by using a voice command, a specific gesture, or a control button, as used in the usability test. The POI information outside looks the same in regular mode and convoy mode.


Figure 7. Function of pinning vessels by gazing.

In both modes, the navigator may gain access to the icebreaker assistance UI when moving around on the bridge. The application can be independently displayed in AR while looking backward from the aft bridge (Figure 8). As exemplified in Figure 8, which is a photo mockup designed to illustrate how the UI would appear in use, exploring areas without function, such as window bars, can be useful.

With the Icebreaker Assistance application, we see several possible UI representations in AR that can be adapted to the situation in a seamless way that can support a navigator's situational awareness. However, to begin validating the application proposal as a concept, we chose to evaluate the proposals described above in a usability test.

\subsection{Usability Test of the Application}

In the following section, we present a usability study of the current versions of the design proposal for the Icebreaker Assistance application, conducted in a virtual demonstrator by professional mariners. The results are presented from both the thinkaloud protocol and the questionnaire. 


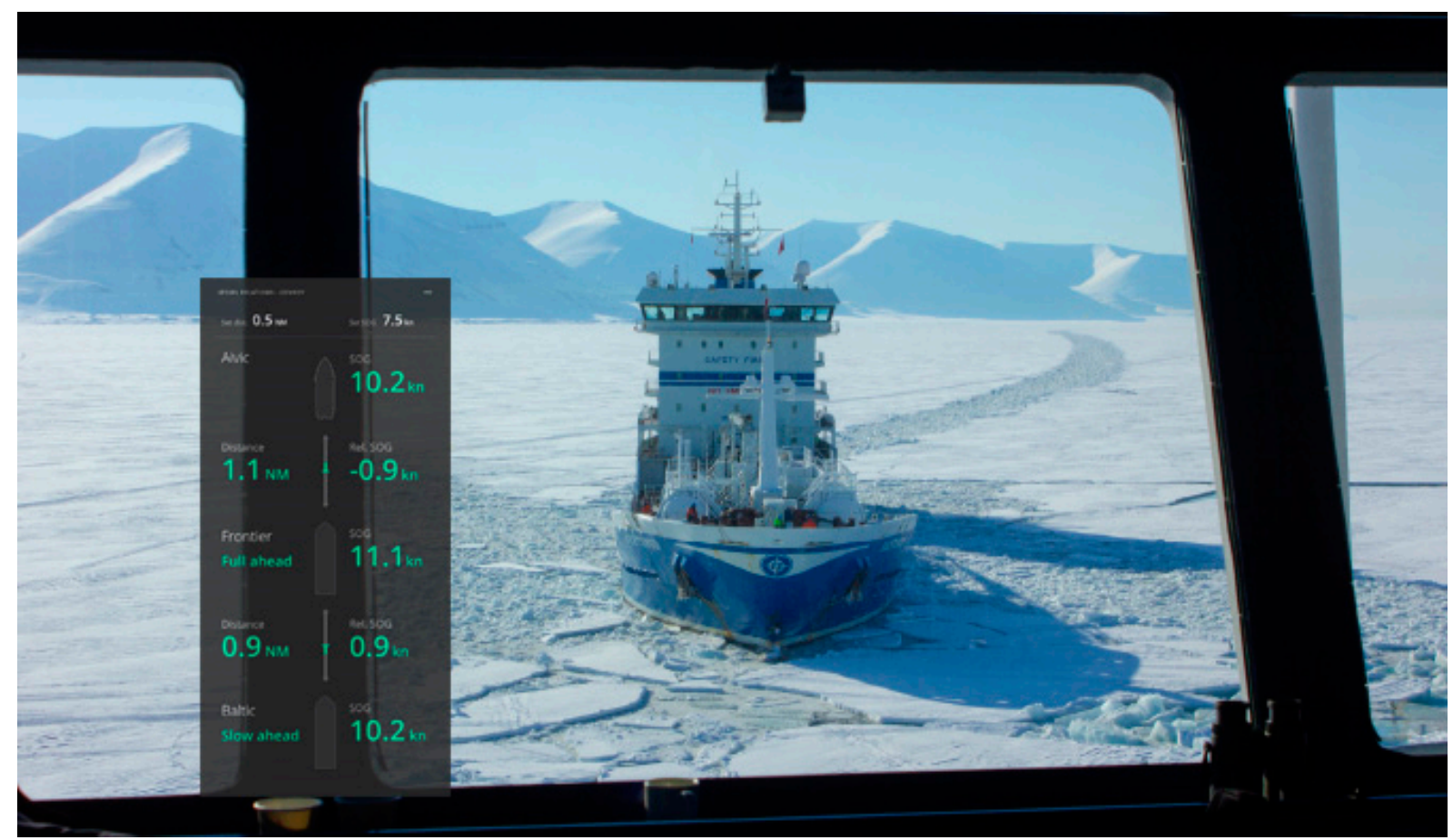

Figure 8. Use of convoy mode in Icebreaker Assistance separately displayed on the aft bridge in a photo-realistic mockup.

\subsubsection{Results of the Think-Aloud User Test}

Tables 2-4 summarize the results from the concurrent think-aloud protocol.

Table 2. Participant feedback from the UI setup (information pane).

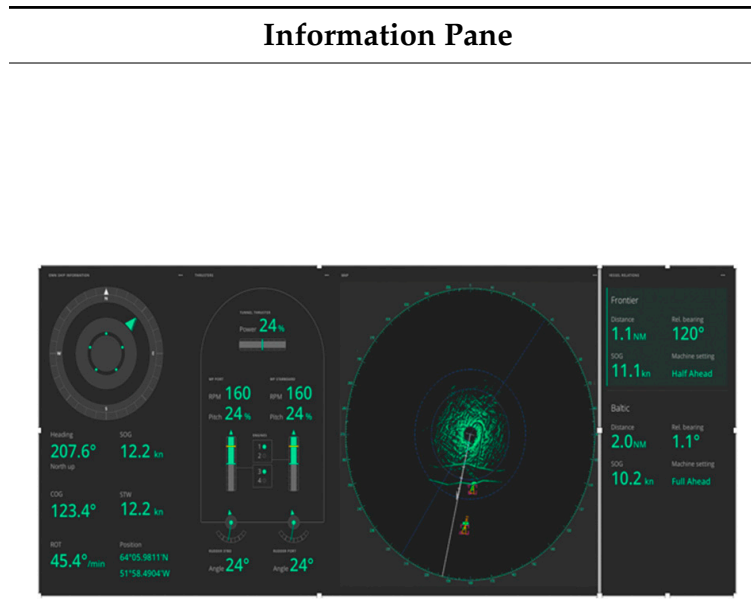

\section{Participant Feedback}

Participants like that the information pane moved with them around the bridge. This could improve SA.

Participants liked having the radar on the panel, this was very useful for ice operations.

Panel was intuitive and clear and had useful information and a good color scheme.

Participants suggested to not add any more information to this panel, as this could risk information overload.

Opinions differed on what information is the most important and where it should be placed. In general, participants liked the way it looked.

Participants liked the ability to switch between VRM and convoy mode.

Operator should have the opportunity to customize the information on the panel by choosing what information is visible and where it is placed on the panel.

Participants suggested slightly increasing the size of the labels (primarily to the left of the engine panel).

Participants suggested slightly lowering the placement of the panel. They noted the possibility of having neck pain from looking up too much. 
Table 3. Participant feedback from POI appearance and placement.

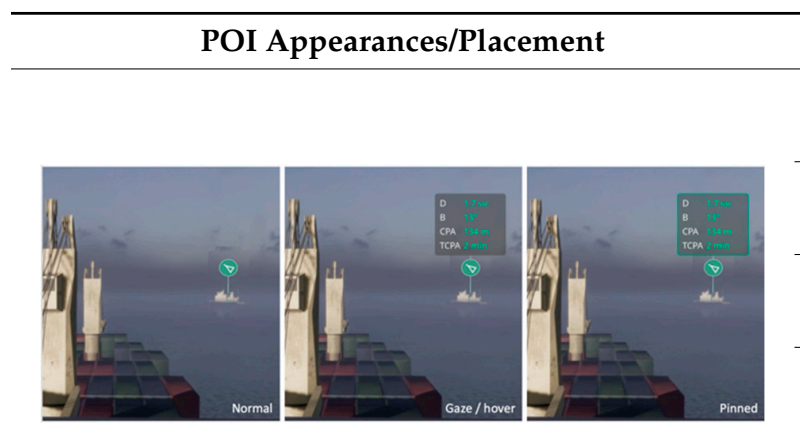

Participant Feedback

POI information panel had relevant information, and the participants liked that the panel could be locked and was visible "through the walls".

POI changing colors made sense, but the arrow was not intuitive. What the arrow was pointing at and why was difficult to interpret.

Participants suggested adding speed to the information panel above the POI, which was agreed to be the most crucial information for this operation.

Participants suggested adding more information about the vessel which the operator could choose to see (e.g., speed, name of the vessel [AIS information]).

When the vessels were close to each other, the panels overlapped, which sometimes caused confusion.

Table 4. Participant feedback on the vessel relations and convoy modes.

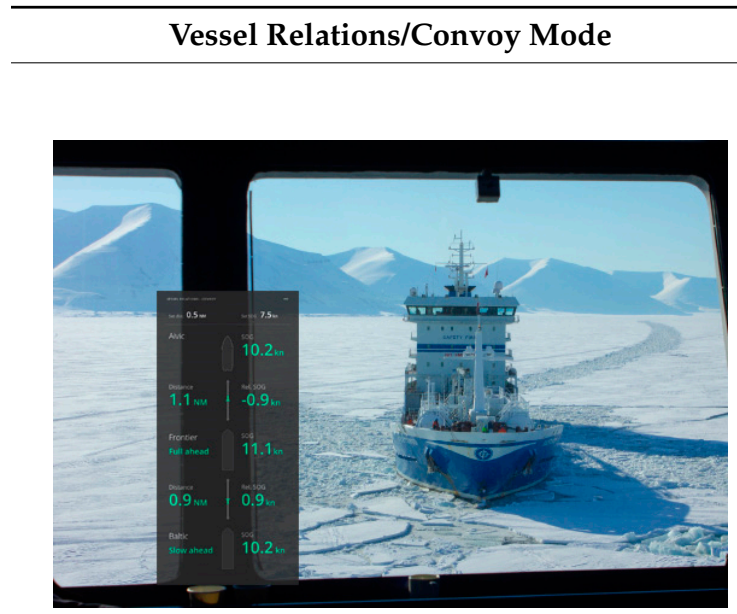

\section{Participant Feedback}

Extremely useful and intuitive function in a convoy situation. This will help with the communication of important information to other vessels.

Participants would like this convoy panel to be available at all places on the bridge and to be able to put it up when needed.

Arrows in the middle with the small green symbol were very hard to see.

Participants suggested to change their color or enlarge and clarify them.

The idea is good but difficult to understand, given the small size.

Participants suggested a color change in the presence of "danger;" if the

vessel POI is red, perhaps the information in the panel/convoy mode should also be red, yellow, etc., to align both images together.

Participants suggested not adding any more information to this panel to avoid overcrowding.

Having machine settings in this panel is very valuable. Obtaining accurate information is currently difficult, as it is mostly from observing the other vessel.

\subsubsection{Results from the Retrospective Verbal Reports}

The following list provides a summary of the retrospective reports from the participants. This was collected after the participants completed the scenario and had a chance to reflect on the experience and provide a higher-level perspective of the purpose and use of these solutions in icebreaker and convoy operations:

- Overall, the participants had a positive attitude toward the AR solutions and observed clear benefits for its use in a convoy scenario in ice-covered waters;

- The participants believed that these solutions could improve safety in icebreaker and convoy operations;

- The participants strongly advocated that these types of solutions must be flexible and customizable for individual operators and for specific operations (e.g., convoy). Convoy operations require the navigator to constantly look out the windows at other ships and down at the ice to observe the distances between vessels. The only information that should be presented during this part of the operation is data that can help the operator safely complete the operation. When the vessels are lined up in the convoy, an option to obtain the most important information should be available;

- The participants agreed that this technology could improve SA. This concept allowed them to maintain a heads-up position to monitor the outside world, paired with important vessel information, which was more integrated than in real life;

- Several participants mentioned the importance of avoiding information overload. This was generally tied to the ability to turn the AR widgets on/off when needed; 
- All participants agreed that the standardized design of the navigational information (OB UI) used in the test was positive and should be implemented on board;

- The participants brought up interesting aspects of how this technology and test setup could be used for training, particularly for less experienced operators. Given the complexity of convoy operations on ice, using VR as a tool to provide cadets with exposure to this type of situation would be very beneficial.

\subsubsection{Results from the Post-Test Questionnaire}

In terms of the overall experience, five participants reported "very positive", and two reported "positive" on a five-point scale from "very negative" to "very positive". When asked "What is your attitude toward the value of using AR solutions for navigation?" in the pre-test questionnaire, the participants responded very positively both pre- and post-test. Only one participant changed their perspective on the value of AR solutions after testing them toward a more positive attitude. Six out of seven participants believed that AR solutions were either very valuable or extremely valuable for navigation, and one participant believed they were somewhat valuable. This is to be expected, given the already positively skewed responses prior to testing the AR solutions.

Figure 9 provides a summary of the results of the remaining five questions in the post-test questionnaire. The results show a positive experience and attitude toward the use and application of the AR UI. Only one participant did not agree that there is a clear benefit to using AR solutions for navigation. Additional comments provided by the participant indicate that their hesitancy stems from the possibility of over-reliance on the AR information and the potential for information overload.

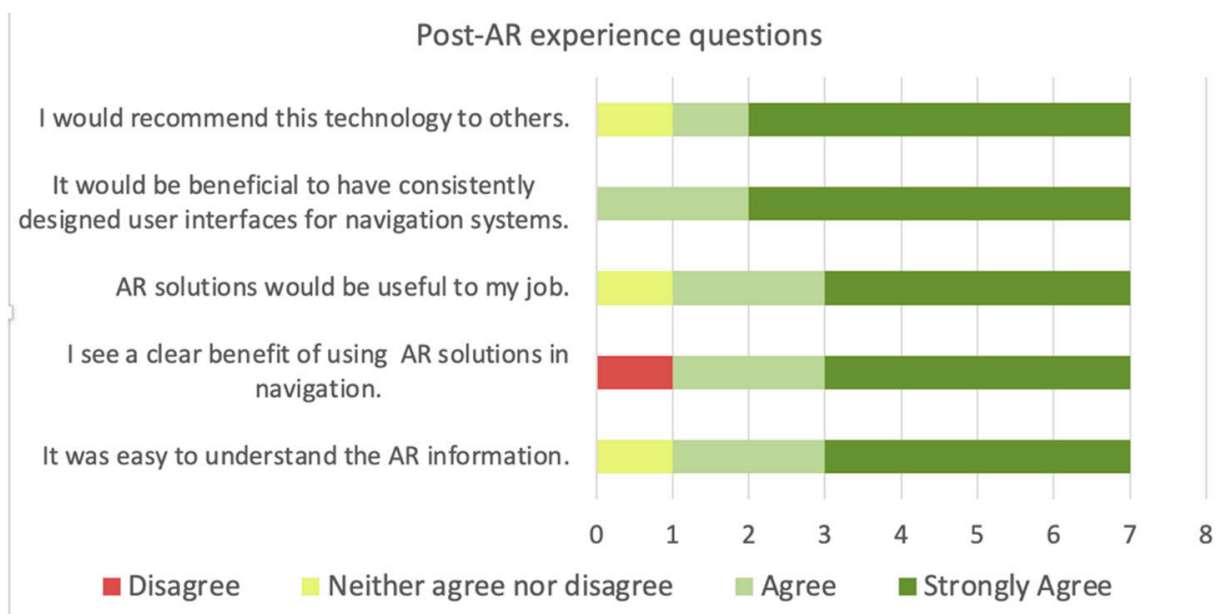

Figure 9. General questions about AR solutions that were part of the post-AR experience.

\section{Discussion}

In this paper, we present a concept showing how an AR UI can be designed to support icebreaker and convoy operations in ice. Although the usability study shows promising results, the application concept is premature from a design for new technology perspective, and further research is needed to determine if the app concept can actually support icebreaker operations by improving usability, efficiency, and safety. However, the rationale behind this study is to develop an evaluable example. In the following sections, we discuss some quality factors that we believe are important to highlight in regard to the research question.

Coordinated vessel data are an important usability issue. Relevant data from several vessels in a uniform UI setup make it easier to monitor all ships at the same time. The navigator will be spared from having to do demanding integration work between systems to compare key data, such as speed, heading, and propulsion. An icon representation of the vessels in the correct order makes the UI comparable to a real-world situation. The 
relative values between each vessel are a new parameter that the application offers, which navigators must manually calculate today. Furthermore, a coordinated application, such as icebreaker assistance, can reduce the need for verbal communication between the icebreaker navigator and the supported vessels. Today, the primary mode of communication is via VHF radios, even though they are associated with a high risk of miscommunication and misunderstanding [40]. The proposed application can transmit key data, information, and commands, thereby reducing the risk of misunderstandings and improving communication between team members to help obtain shared situation awareness. A shared SA can be defined as a shared understanding of a situation among team members at one point in time [41].

A convoy scenario is a specific type of operation that could directly benefit from coordinated vessel data and improved shared SA. As an example, during user testing, one of the participants who had extensive real-world experience in ice navigation spent most of the time looking out the bridge wing windows away from the main control station. He indicated that experienced ice navigators focus on the visuals, feel of the vessel, feedback from the engines, and ice movements according to ship movements. Normally, this navigator would rely on their colleagues to report the numerical information necessary to fully understand the situation while also clearly reporting their personal understanding of the situation. This information loop is at risk of miscommunication and misunderstanding. The application would allow the navigator to stand on the bridge wing to look out the window while also being able to see the status and distance to nearby vessels in all situations when multiple vessels are close to each other, along with others present on the bridge. In addition, we propose that all the vessels in the convoy should have access to the application and the coordinated vessel data, which would further promote a shared SA between all vessels and increase the transparency of decision making.

Situation-based UIs have the potential to improve usability. The concept demonstrates a situation-based adaptation of the application by dividing it into two different modes, representing the stages of the operation. We suggest that the modes are manually set by the user. The number of modes could potentially be increased if user needs in the different stages of the operation proved to be remarkably different. We also suggest that modes for critical stages in an icebreaker operation, such as breaking another vessel free from ice, might need an individual mode with a UI setup adapted to high levels of stress. This implies removing unnecessary information and perhaps enabling multiple output modes, such as voiceover for critical data. We suggest that designing applications with several situational modes for complex and sequence-based operations, such as icebreaker assistance, can be a way to support the operation and increase safety. The application also adapts to the user situation by offering different UI representations adapted to different user zones in the bridge. User zones, also referred to as AR zones, refer to a sectioning of the bridge (or workspace) based on physical, spatial, and conditional characteristics, such as poor or good view of a certain element, and user needs, such as the need to monitor another system or handling devices [4].

What impact on safety can applications, such as Icebreaker Assistance, have? The maritime industry is technology driven [42], and ensuring that new technology will support the operator rather than introduce unwanted risk is important. When asked about safety, the participants agreed that highlighting various types of critical operational information at the right time could improve safety. Furthermore, the participants believed that the UI concept could improve their SA through the ability to maintain a consistent lookout while having access to critical information. When asked if these UI concepts could reduce the risk of a convoy-related accident, six out of seven participants agreed that the risk of an accident would be reduced, and one participant was torn between the benefits and risks of the technology.

Identifying the risks is important, given that integrating AR technology in maritime operations has potential safety concerns. This matter was one of the post-test questions that was discussed with the participants. In general, the participants raised concerns about 
the possibility of information overload, especially in high-traffic areas. They indicated that the only way that this technology could be used safely is for the user to have the ability to turn it on and off, and the ability to remove the headset whenever needed.

Furthermore, some of the UI representations caused confusion for the participants. One example was the POI arrow symbol indicating another ship (Figure 6). All participants had varying ideas of what this represented and determined that it was not easy to understand, which could lead to a risk of distraction. This reiterates the importance of user testing and user involvement in the early stages of the design process for the application to be able to quickly adapt solutions to user needs [23]. Finally, in some parts of the scenario, the information overlapped, which the participants noted as something that should be avoided as much as possible. Displaying one information layer at a time should be possible to reduce confusion. Overall, these safety concerns are minor and can easily be adapted in the design phase through the feedback process adopted in this study. New technology always poses risks; however, the potential safety benefits of this technology seem to outweigh its potential risks.

Several limitations have affected the method. User testing was completed during the COVID-19 pandemic. This caused adaptations to the test protocol, including safe distancing and increased sanitation practices. Furthermore, COVID-19 restrictions caused a reduction in participant availability, resulting in a relatively small and homogeneous sample size. However, for usability studies, five test persons have been cited as enough to find most of the usability issues with a product, with any more leading to an observation of repeated results $[43,44]$. Data saturation was experienced during the final stages of the scenario testing, which led the researchers to finalize the data collection. Although it is a widely used method, the think-aloud protocol has some limitations. This method has been criticized for its ability to capture participants' genuine thoughts about the activity, given individual personality traits, and the ability to verbalize thoughts in a research setting [37]. An additional challenge with this method is ensuring that participants feel comfortable being honest and potentially critical about their reflections. Although challenging, the test setup and participant group in this study decreased the influence of these limitations. The participants in this study were seafarers who were trained in talking through their thought process for decision making in critical situations, meaning the think-aloud protocol was somewhat natural for them. Furthermore, the distribution of the research team between Oslo and Gothenburg allowed the HF specialists conducting the test and the AR architects and designers to operate independently, which was clearly communicated to the participants. We believe that this setup helped the participants feel comfortable in criticizing the scenario, as the researchers who were present at the data collection were not responsible for developing the solutions.

Another important issue we would like to bring into the discussion is the effect that a consistent UI architecture has on usability. The UI concept presented is designed to be a part of a consistent UI design for all ship bridge workplaces. Design consistency is an important quality for UIs because it improves a user's ability to switch and transfer competence, logic, and skills between different UI units and across various systems [45]. Given that AR equipment will be used with existing workplaces, we believe that it should share characteristics with other interfaces on the ship's bridge. To achieve such consistency, we built the AR UI on the $\mathrm{OB}$ design system, an open-source platform that enables various vendors to develop cost-effective, safe, and efficient UIs for the maritime domain. One of the core goals of OB is to achieve improved design consistency across a workplace assembled by systems from multiple vendors [39]. The design system is in continuous incremental development through several industry-driven research projects. This form of the standardization of navigational equipment seems to show obvious advantages for both vendors and users, as the registration rate for using the $\mathrm{OB}$ system is continuously increasing.

The AR interface development has adapted components for $\mathrm{OB}$ to $\mathrm{AR}$ and produced results that will be used to extend $\mathrm{OB}$ guidelines to cover AR applications. This is an important contribution, given that limited precedence exists in research and practice on 
how to design AR interfaces for ships. The proposed design cases will help build better knowledge of AR solution space for maritime applications.

Furthermore, the use of VRROS for concept development and testing is a novel approach for design development. In this study, AR UIs were realized in VR to develop and test AR concepts. Interpreting and adapting the OB design system to work for AR is challenging, both in terms of development and testing, for several reasons. Accessibility to the user context is limited. It is highly necessary to conduct field work to develop and test AR prototypes, given that they closely connect to the physical context. The use context has rapidly changing conditions, which alter the premises for design. Finally, conducting design processes within the user context is very demanding.

The VRROS provide the opportunity to overcome several of these challenges with its easy access and manipulatable conditions, and a far less demanding environment to design within. In addition, access to perform usability testing had a far lower threshold than testing in real conditions. The methodological approach of performing virtual fieldwork through the use of VRROS was also tested on master's students at AHO due to COVID-19 measures in Autumn 2020 and tends to have high efficiency for quick prototyping and evaluation of UI ideas. Despite the efficiency of virtual fieldwork and testing, we would like to emphasize that we believe that development and usability testing in a real-world context would most likely have revealed several other relevant requirements and problems with the design that needed to be tackled. As such, we see the VRROS approach as a complementary addition to real-world design development and usability testing.

Throughout the testing, the participants were asked about their experience of testing AR UIs in VR. Most of the participants were skeptical at first, given their limited knowledge of and exposure to VR technology. However, once acclimated to the VR environment, all the participants recounted a positive experience, with no feelings of malaise at any point throughout the study. This resulted in a high level of user acceptance of VR to evaluate the UI concepts. In addition, every participant commented on the high-quality visuals and overall realistic feeling of being on the bridge. Immersive technologies, including virtual reality, AR, and mixed reality, are becoming more viable options for maritime education and training [46]. This study provided a positive experience, and as the technology improves, immersive technologies will likely become even more affordable and available for maritime research.

Regarding further development of the concept, the Icebreaker Assistance app concept is incomplete, and several aspects need to be explored, defined, and further developed. Some examples that are planned to be further developed include the activation of convoy mode; a convoy planning tool; distributed representation of data, alerts, and notifications; the use of the application in other situations.

\section{Conclusions}

In this article, we present a design concept and usability study of an AR application called Icebreaker Assistance, which supports convoy operations on icebreaker vessels. The work adopted new VR scenario-driven design and test methods. The purpose of the study was to explore and evaluate how AR UIs can be designed to support icebreaker operations and improve navigator safety. We demonstrated and user-tested a novel UI design that adapts the UI representations according to where the user moves on the bridge.

The AR concept tested in this study shows promising potential to improve safety during convoy operations in Arctic waters. The AR concept has the ability to integrate currently distributed information, decrease the chance of miscommunication within and between vessels, and possibly improve operator SA. The participants remained cautiously optimistic about the further development of AR UIs, indicating that the potential risks of information overload and distracting visuals should not be underestimated. This study presents an initial concept development and small-scale usability study that can be used to establish a foundation for evaluation and further research. 
Author Contributions: Conceptualization, J.O.H.E., K.N. and S.F.; methodology, S.F., K.A., K.N. and J.O.H.E.; software, J.O.H.E.; validation, J.O.H.E., K.N.; formal analysis S.F., J.O.H.E., K.N., K.A.; investigation, J.O.H.E., S.F., K.A. and K.N.; resources, K.N.; data curation, K.A.; writing-original draft preparation, S.F., K.A.; writing-review and editing, K.N., J.O.H.E.; visualization, J.O.H.E.; supervision, K.N.; project administration, K.N.; funding acquisition, K.N. All authors have read and agreed to the published version of the manuscript.

Funding: The research presented in this paper was funded by the EU project SEDNA. This project receives funding from the European Union's Horizon 2020 research and innovation program under grant agreement no. 723526 .

Institutional Review Board Statement: Not applicable.

Informed Consent Statement: Informed consent was obtained from all the subjects involved in the study.

Acknowledgments: We would like to thank the project team of the EU project SEDNA, who have provided valuable knowledge and contributions in realizing the design concepts and usability testing across several campuses. We also give thanks to the participants in the user test who shared their knowledge and experience in their evaluations.

Conflicts of Interest: The authors declare no conflict of interest. The funders had no role in the design of the study; in the collection, analyses, or interpretation of data; in the writing of the manuscript; or in the decision to publish the results.

\section{References}

1. Grabowski, M.; Rowen, A.; Rancy, J.-P. Evaluation of wearable immersive augmented reality technology in safety-critical systems. Saf. Sci. 2018, 103, 23-32. [CrossRef]

2. Frydenberg, S.; Eikenes, J.O.; Nordby, K. Serendipity in the field. Facilitating serendipity in design-driven field studies on ship bridges. Des. J. 2019, 22, 1899-1912. [CrossRef]

3. Frydenberg, S.; Norby, K.; Eikenes, J.O. Exploring designs of augmented reality systems for ship bridges in arctic waters. In Proceedings of the Rina-International Conference on Human Factors, London, UK, 26-27 September 2018.

4. Nordby, K.; Etienne, G.; Frydenberg, S.; Eikenes, J.O.H. Augmenting OpenBridge: An open user interface architecture for augmented reality applications on ship bridges. In Proceedings of the Computer Applications and Information Technology in the Maritime Industries, COMPIT'20, Online Conference, 9-10 August 2020.

5. Luras, S.; Nordby, K. Shaping designers' sea sense: A guide for design-driven field research at sea. In Proceedings of the Marine Design 2015, London, UK, 2-3 September 2015.

6. Giacomin, J. What is human centred design? Des. J. 2014, 17, 606-623. [CrossRef]

7. Laera, F.; Fiorentino, M.; Evangelista, A.; Boccaccio, A.; Manghisi, V.M.; Gabbard, J.; Gattullo, M.; Uva, A.E.; Foglia, M.M. Augmented reality for maritime navigation data visualisation: A systematic review, issues and perspectives. J. Navig. 2021, 74, 1073-1090. [CrossRef]

8. Røds, J.-F. Navitagtion in the Arctic. How Can Simulator Training be Used for Assessment and Reduction of Risk? The Arctic University of Norway: Tromsø, Norway, 2018.

9. Rogers, D.D.; King, M.; Carnahan, H. Arctic search and rescue: A case study for understanding issues related to training and human factors when working in the north. In The Interconnected Arctic-UArctic Congress 2016; Springer Science and Business: Berlin/Heidelberg, Germany, 2020; pp. 333-344.

10. Topaj, A.; Tarovik, O.; Bakharev, A.; Kondratenko, A. Optimal ice routing of a ship with icebreaker assistance. Appl. Ocean Res. 2019, 86, 177-187. [CrossRef]

11. Rosenblad, M. Increasing the Safety of Icebound Shipping-WP4 Operative Environment (Icebreaker Operations); Helsinki University of Technology: Espoo, Finland, 2007.

12. Banda, O.; Goerlandt, F.; Montewka, J.; Kujala, P. Winter navigation at the Baltic Sea: An analysis of accidents occurred during winters 2002-2003 \& 2009-2013. In Safety and Reliability, Methodology and Applications, 1st ed.; Nowakowski, T., Mlynczak, M., Jodejko-Pietruczuk, A., Werbinska-Wojciechowska, S., Eds.; Taylor \& Francis Group: London, UK, 2015; pp. 83-92. [CrossRef]

13. Goerlandt, F.; Montewka, J.; Zhang, W.; Kujala, P. An analysis of ship escort and convoy operations in ice conditions. Saf. Sci. 2017, 95, 198-209. [CrossRef]

14. Buitendijk, M. Assumptions and Poor Communication Lead to Vessel Collision SWZ Maritime. SWZ Maritime. Available online: https:/ / www.swzmaritime.nl/news/2021/03/10/assumptions-and-poor-communication-lead-to-vessel-collision/ (accessed on 10 June 2021).

15. Oconnor, P. Assessing the effectiveness of bridge resource management training. Int. J. Aviat. Psychol. 2011, 21, 357-374. [CrossRef] 
16. Yastrebova, A.; Hoyhtya, M.; Boumard, S.; Lohan, E.S.; Ometov, A. Positioning in the arctic region: State-of-the-art and future perspectives. IEEE Access 2021, 9, 53964-53978. [CrossRef]

17. Kongsberg, M. Laser-Based Position Reference System. Available online: https://www.kongsberg.com/no/maritime/products/ vessel-reference-systems/position-systems/laser-based-position-reference-system/ (accessed on 14 June 2021).

18. Kongsberg, M. Differential Absolute and Relative Position Sensor, DARPS. Available online: https://www.kongsberg.com/no/ maritime/products/vessel-reference-systems/position-systems/differential-absolute-relative-position-sensor/ (accessed on 14 June 2021).

19. Endsley, M.R. Toward a theory of situation awareness in dynamic systems. Hum. Factors J. Hum. Factors Ergon. Soc. 1995, 37, 32-64. [CrossRef]

20. Jussila, J.; Lehtonen, T.; Laitinen, J.; Makkonen, M.; Frank, L. Visualising maritime vessel open data for better situational awareness in ice conditions. In Proceedings of the 22nd International Academic Mindtrek Conference, New York, NY, USA, 10-11 October 2018. [CrossRef]

21. Lützhöft, M.; Nyce, J. Integration work on the ship's bridge. J. Marit. Res. 2008, 5, 59-74.

22. Microsoft HoloLens. Mixed Reality Technology for Business. Available online: https://www.microsoft.com/en-us/hololens (accessed on 21 June 2021).

23. Gernez, E. Human-Centered, Collaborative, Field-Driven Ship Design: Implementing Field Studies for The Design of Ships in Operation. Oslo School of Architecture and Design. Available online: https://aho.brage.unit.no/aho-xmlui/handle/11250/2608 505 (accessed on 8 June 2021).

24. Baldauf, M.; Procee, S. Augmented reality in ships bridge operation. In Proceedings of the ISIS 2014-International Symposium Information on Ships, Berlin, Germany, 27-28 September 2018. Available online: https://www.researchgate.net/publication/27 3057248_Augmented_Reality_in_Ships_Bridge_Operation (accessed on 13 April 2018).

25. Hareide, O.S.; Porathe, T. Maritime augmented reality. Coord. Mag. 2019, 15, 31-35.

26. Procee, S.; Borst, C.; Van Paassen, R.; Mulder, M. Using augmented reality to improve collision avoidance and resolution. In Proceedings of the 17th International Conference on Computer and IT Applications in the Maritime Industries, Pavone, Online Conference, 28-30 October 2021. Available online: https:/ / repository.tudelft.nl/islandora/object/uuid\%3A42f31d3a-d18b-41b9 -9b09-bf1c0643a96c (accessed on 17 February 2021).

27. Aylward, K.; Dahlman, J.; Nordby, K.; Lundh, M. Using operational scenarios in a virtual reality enhanced design process. Educ. Sci. 2021, 11, 448. [CrossRef]

28. Nordby, K. OpenBridge Design Guideline. Available online: http://www.openbridge.no/guideline.html (accessed on 22 February 2021).

29. Siang, T. What is Interaction Design? The Interaction Design Foundation. 2018. Available online: https://www.interactiondesign.org/literature/article/what-is-interaction-design (accessed on 28 June 2021).

30. Luras, S.; Nordby, K. Field Studies Informing Ship's Bridge Design at the Ocean Industries Concept Lab. In Proceedings of the Human Factors in Ship Design \& Operation, RINA, London, UK, 26-27 February 2014.

31. De Walt, K.M.; De Walt, B.R. Participant Observation: A Guide for Fieldworkers; Rowman Altamira: Lanham, MD, USA, 2011.

32. Sanders, E.B.-N.; Stappers, P.J. Co-creation and the new landscapes of design. CoDesign 2008, 4, 5-18. [CrossRef]

33. Hareide, O.S.; Ostnes, R. Maritime usability study by analysing eye tracking data. J. Navig. 2017, 70, 927-943. [CrossRef]

34. Lurås, S.; Lützhöft, M.; Sevaldson, B. Meeting the complex and unfamiliar: Lessons from design in the offshore industry. Int. J. Des. 2015. Available online: http:/ /www.ijdesign.org/ojs/index.php/IJDesign/article/view/1845 (accessed on 21 September 2017).

35. Eikenes, J.O. How to Create Your own HoloLens Sun-Screen by Jon Olav, H. Eikenes. Ocean Industries Concept Lab Medium. Available online: https:/ / medium.com/ocean-industries-concept-lab/how-to-create-your-own-hololens-sun-screen-68c466 071a01 (accessed on 8 June 2021).

36. Etikan, I.; Musa, S.A.; Alkassim, R.S. Comparison of convenience sampling and purposive sampling. Am. J. Theor. Appl. Stat. 2016, 5, 1-4. [CrossRef]

37. Fan, M.; Shi, S.; Truong, K.N. Practices and challenges of using think-aloud protocols in industry: An international survey. J. Usability Stud. 2020, 15, 85-102.

38. SurveyMonkey. SurveyMonkey: The World's Most Popular Free Online Survey Tool. Available online: https://www. surveymonkey.com/ (accessed on 21 June 2021).

39. Nordby, K.; Frydenberg, S.; Fauske, J. Demonstrating a maritime design system for realising consistent design of multi-vendor ship's bridges. In Proceedings of the Conference on Human Factors, Orlando, FL, USA, 21-25 July 2018.

40. Aylward, K.; MacKinnon, S.N.; Lundh, M. Preventing unruly technologies in maritime navigation: A systems approach. In Advances in Intelligent Systems and Computing; Springer Science and Business: Berlin/Heidelberg, Germany, 2020; pp. 421-428.

41. Salas, E.; Prince, C.; Baker, D.P.; Shrestha, L. Situation awareness in team performance: Implications for measurement and training. Hum. Factors J. Hum. Factors Ergon. Soc. 1995, 37, 123-136. [CrossRef]

42. Aylward, K.; Johannesson, A.; Weber, R.; MacKinnon, S.N.; Lundh, M. An evaluation of low-level automation navigation functions upon vessel traffic services work practices. WMU J. Marit. Aff. 2020, 19, 313-335. [CrossRef]

43. Nielsen, J.; Landauer, T.K. Mathematical model of the finding of usability problems. In Proceedings of the INTERACT'93 and CHI'93 Conference on Human Factors in Computing Systems, Amsterdam, The Netherlands, 24-29 August 1993; ACM: New York, NY, USA, 1993; pp. 206-213. [CrossRef] 
44. Nielson, J. Why You Only Need to Test with 5 Users; Nielsen Norman Group: Fremont, CA, USA, 2000. Available online: https://www.nngroup.com/articles/why-you-only-need-to-test-with-5-users/ (accessed on 15 November 2020).

45. Nielsen, J. Coordinating user interfaces for consistency. ACM SIGCHI Bull. 1989, 20, 63-65. [CrossRef]

46. Mallam, S.C.; Nazir, S.; Renganayagalu, S.K. Rethinking maritime education, training, and operations in the digital era: Applications for emerging immersive technologies. J. Mar. Sci. Eng. 2019, 7, 428. [CrossRef] 